\title{
Wegener Granulomatosis: Case Report of Facial Prosthetic Rehabilitation
}

\author{
Simone Cristina Kawabata ${ }^{a}$, Patricia Tiemy Hirono Hotta ${ }^{a}$, Reinaldo Brito e Dias ${ }^{\mathrm{a}}$, \\ Beatriz Silva Camara Mattos ${ }^{\mathrm{a}, \mathrm{b}}$
}

\begin{abstract}
Wegener granulomatosis (WG) is a systemic, autoimmune disease histologically characterized by granulomatous vasculitis that affects the upper and lower aerodigestive tracts and glomerulonephritis. The purpose of this study was to restore the esthetic aspect of a saddle nose modified by WG. The clinical case is on a prosthetic rehabilitation in a 53-year-old woman with a severe nose deformity resulting from WG. The sculpture was made in accordance with the requests expressed by the patient and within the limits of the working area. A medical silicone, Silastic MDX-4-4210, was used to make the prosthesis in order to ensure adequate fitting and esthetic appearance. The rehabilitation provided by the maxillofacial prosthesis raised the patient's self-esteem and caused an improvement in life quality leading to the social reintegration. Dentists must be aware of oral signs such as strawberry gingivitis, which may arise early and is a pathognomonic sign of the disease. Maxillofacial prosthesis may be the only esthetic solution in cases of saddle nose due to in cases of WG.
\end{abstract}

Keywords: Wegener granulomatosis; Maxillofacial prosthodontics; Nasal prosthesis

\section{Introduction}

Wegener granulomatosis (WG) was first described by Friedrich Wegener in 1936 [1]. The disease, currently known as granulomatosis with polyangiitis (GPA) [2, 3], is a comparatively rare systemic autoimmune disorder of unknown etiology [4]. When characterized by the triad of granulomatous inflammation, necrosis and vasculitis (inflammation of small and medium arteries and veins) that affects the upper and lower airways and kidneys, it is known as generalized [2] and when

Manuscript accepted for publication November 17, 2014

aDepartment of Surgery, Prosthesis and Maxillofacial Traumatology, Dental School, University of Sao Paulo, Sao Paulo, Brazil

${ }^{b}$ Corresponding Author: Beatriz Silva Camara Mattos, Dentistry School, University of Sao Paulo, Professor Lineu Prestes Avenue, 2227, 05508-000 Sao Paulo, SP, Brazil. Email: bscmatto@usp.br

doi: http://dx.doi.org/10.14740/jmc2028e it only affects the lungs, it is the limited form of the disease [5]. The most common signs and symptoms are fatigue, loss of appetite, weight loss, short breath, fever, bloody sputum through the upper airways, joint pain, sinusitis, skin nodules and ulcers, eyes and middle ear inflammation, as well as neuropathy [5]. The nonspecific manifestations may cause the diagnosis of this disease to be hard and particularly complex, thereby delaying the appropriate treatment determination [6]. As a consequence, sequel arises due to tissue degeneration of the affected area.

The prevalence of this disease varies from $3 / 100,000$ to $16 / 100,000$ individuals, depending on the location [2]. The syndrome affects men and women regardless of gender, most often observed in individuals between the second and fourth decades [1, 4], as well as the sixth and seventh decades of life [2]. Nevertheless, it can begin at any age $[1,2,4,5]$.

Systemic manifestations reveal the severity of this pathology; pulmonary involvement may range from asymptomatic nodules to pulmonary infiltrates and fulminant alveolar hemorrhage. Segmental necrotizing glomerulonephritis and proliferative glomerulonephritis are the most common renal manifestations [2]. GPA can also affect many other organs resulting in changes in joint, skin (ulcers and subcutaneous nodules) or neurological (mononeuritis multiplex, peripheral neuropathy and meningitis).

Some studies report that GPA is often associated with other clinical disorders in the head and neck (88\%) [2] and they are often the first manifestations of the disease $(28 \%)[2,4]$. Other studies have reported a percentage between $63 \%$ and $72 \%$ [7, 8]. These manifestations may occur in a located area without expression in other organs. These sinus and ear changes are the most frequent. Strawberry gingivitis $[1,6,9]$ in the oral cavity may arise as a rare sign, yet known as a pathognomonic sign of the disease. Gingival hyperplasia, described as erythematous gingivitis, presents widespread pain and easily bleeds when touched.

Clinical abnormalities in the head and neck, associated with GPA, are usually the first manifestations of the disease, being the sinus and otological abnormalities the most frequent ones. Symptoms range from dry nose, purulent nasal discharge, pain, nasal obstruction and olfactory disorders. Nonetheless, from the clinical aspect, there may be crusting of secretions and septal perforation. In severe cases, there are nasal deformities caused by necrosis of the septum, giving rise to the saddle nose. Treatment with corticosteroids and cyclo- 


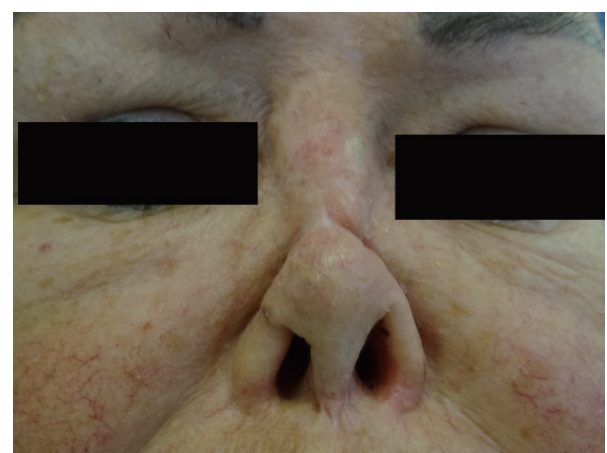

Figure 1. Saddle nose presented by the patient.

phosphamide leads to $90 \%$ remission of the disease within a year. However, if untreated, it leads to death within less than 2 years $[1,2,4,10]$.

Routine laboratory tests are not specific for GPA. Therefore, diagnosis is obtained associating clinical, radiological (multiple pulmonary nodules and sinus), histopathological (granulomatous necrotizing vasculitis and inflammation with the presence of multinucleated giant cells), blood test (neutrophil cytoplasmic antibody (cANCA) positive [5] and inflammatory markers [9]), urinalysis to detect protein and red blood cell [5].

The cANCA is an antibody marker directed to the proteinase 3 (PR3) [9, 10], which exists in azurophilic granules of the neutrophils. Its determination can help the disease diagnosis; however, the positive result is not conclusive and the negative result does not suffice for the exclusion thereof [10]. There are two diagnosis methods: indirect immunofluorescence and enzyme-linked immunosorbent assay (ELISA). Indirect immunofluorescence has specificity of $90 \%$ and the ELISA test has approximately a higher specificity of $98 \%[2,9]$. Although the ELISA test enables a better interpretation, the best choice is to combine both tests.

Some authors indicate the following medications for the disease remission: azathioprine $(2 \mathrm{mg} / \mathrm{kg} / \mathrm{day})$ as safer and as effective as cyclophosphamide, methotrexate $(20 / 25 \mathrm{mg} /$ week), when serum creatinine is less than $1.5 \mathrm{mg} / \mathrm{dL}$, or leflunomide $(20 / 30 \mathrm{mg} /$ day $)$ as efficient as methotrexate, but associated with a wider range of adverse effects $[2,9]$.

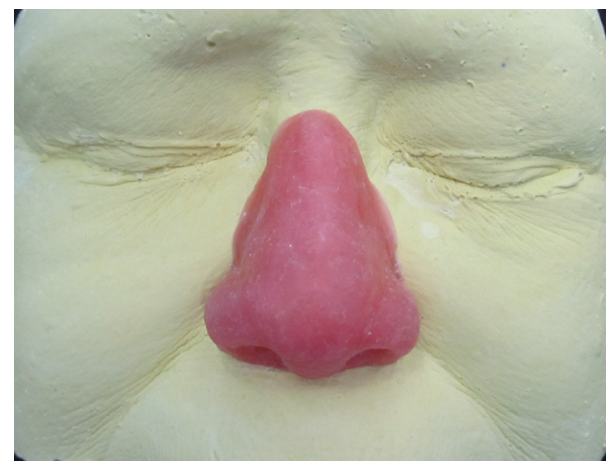

Figure 2. Wax sculpture developed on previously hydrated model.

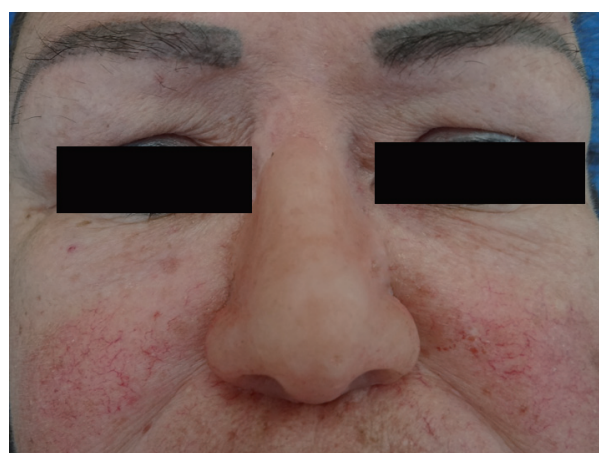

Figure 3. Prosthesis fixed on the face with prosthetic adhesive.

\section{Case Report}

A 53-year-old white woman was admitted to the Maxillofacial Prosthodontics Clinic, Dentistry School, University of Sao Paulo, due to severe nasal deformity (saddle nose), with WG diagnosis (Fig. 1). The patient reported that the first symptom of the disease was observed in the beginning of the third decade of her life with pain and ringing in the ears, which were diagnosed as otomastoiditis and treated surgically. In the same year, two new surgeries were performed, the first one to remove a lump in the left trigeminal nerve, resulting in a condition of trismus, which did not respond to physiotherapy. A second surgery was carried out to improve mouth opening. By 2000 , she started presenting frequent epistaxis with diagnoses of chronic destructive lesion of the median line or WG. The results of histopathological tests indicated no signs of vasculitis, reason why it was hard to reach a diagnosis. Thus, she had 76 sessions of radiotherapy with adjuvant chemotherapy for the

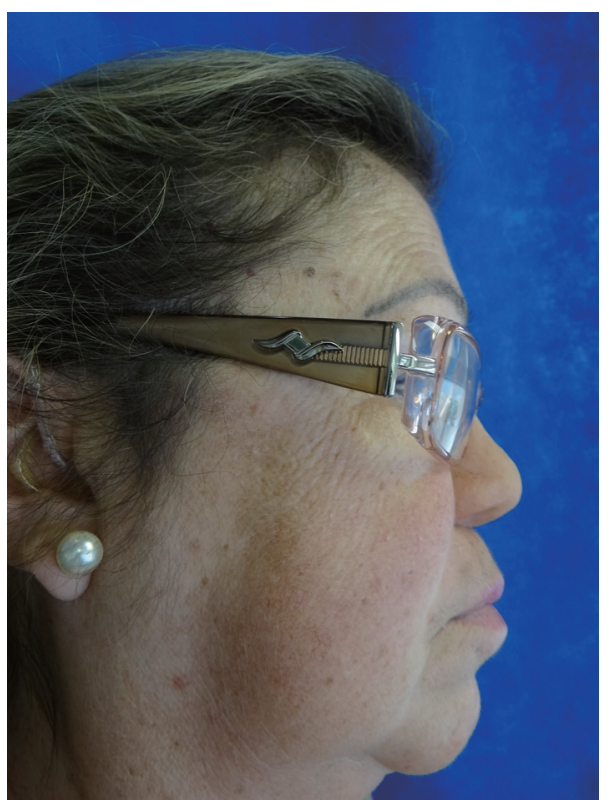

Figure 4. Eyeglasses frame for better concealing the edges of the prosthesis. 


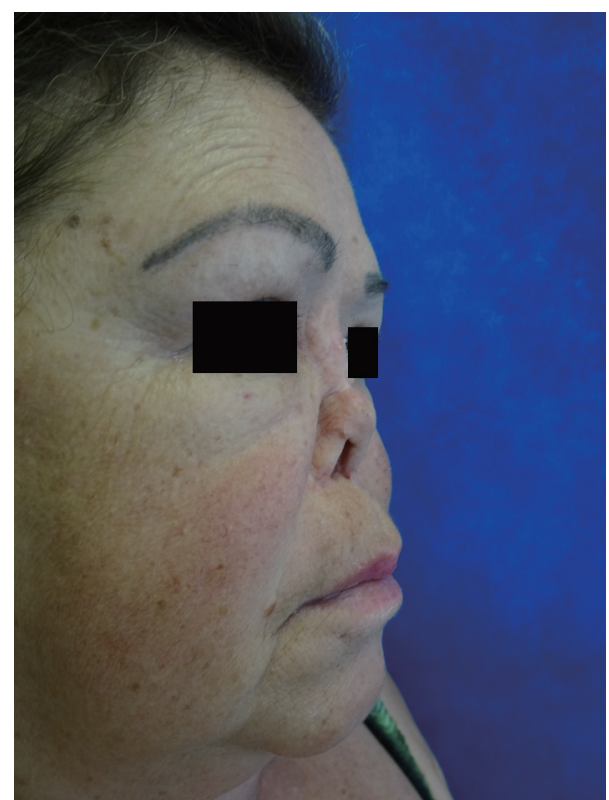

Figure 5. Lateral view of the face without the nasal prosthesis.

treatment of the destructive lesion of the median line and the results thereof were satisfactory.

However, in 2006, she underwent reconstructive graft surgery and had infection in both region, the donor and the receptor area. The biopsies showed signs of vasculitis, finally establishing precise diagnostic of WG. Currently, she is taking prednisone $10 \mathrm{mg} /$ day, $50 \mathrm{mg}$ azathioprine, twice a day, and three varied types of antibiotic with alternated use.

\section{Prosthetic rehabilitation}

The patient sat in a dental chair with a $60^{\circ}$ inclination and no rotation of the head. The moulage impression of the nasal region followed the demarcation: the upper limit was $3 \mathrm{~cm}$ above the glabella, the lower limit was an imaginary horizontal line that ran above the upper lip, and the lateral limit was a vertical line anterior to the tragus. The impression used irreversible hydrocolloid (Jeltrate type II, Dentisply) and the moulage impression was cast in dental stone (Asfer type III, Asfer Ind. Quimica). Therefore, the model obtained allowed working with all the necessary parameters to orient the prosthesis' sculpture.

The sculpture developed on previously hydrated model, using pink wax (Epoxiglass Ind. e Com. de Produtos Quimicos) (Fig. 2) was tested on the face of the patient to a better assessment and guiding of the future prosthesis, in order to reach a consensus between the professional and the patient. The wax model was placed in a no. 6 flask, and silicone MDX 4-4210 (Dow Corning Corporation) was intrinsically characterized with mineral pigments. Various colors were prepared and a small amount of Thixotropic Agent (Factor II) was added so as to enhance the viscosity of the material and reduce its displacement upon pressing.

After polymerization, the prosthesis received the appro-

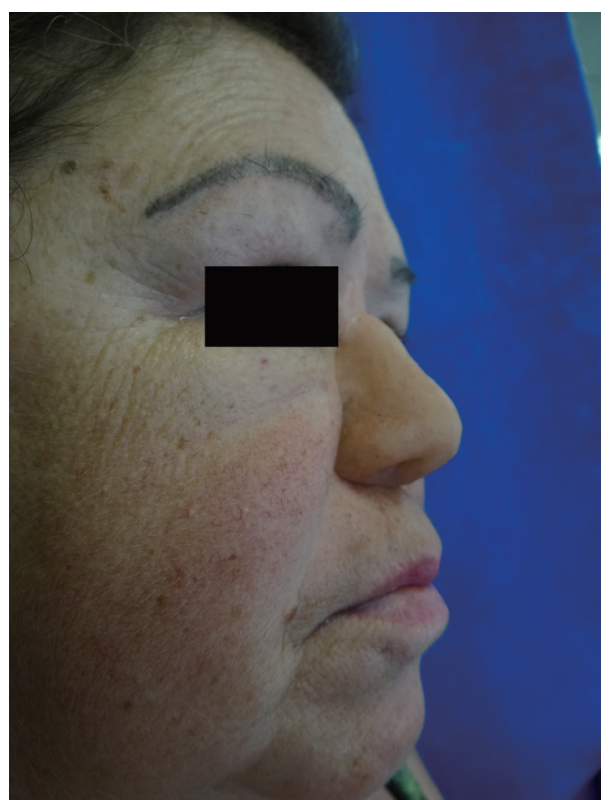

Figure 6. Lateral view of the face with the nasal prosthesis.

priated finishing processes and extrinsic characterization with Extrinsic Coloration System (Factor II) (Fig. 3). It was indicated a fixation with the adhesive Prosthetic Adhesive \#1 (Kryolan Cosmetics) and the use of an eyeglasses frame for better concealing the edges of the prosthesis (Fig. 4).

\section{Discussion}

Aiming at improving the quality of life of patients bearing facial deformities and promoting social rehabilitation, the facial prosthesis improves esthetics and gives comfort to patients. In the present case, despite the remission of the disease, the presence of nasal deformity as a consequence of the disease itself hampers the patient's psychological and social rehabilitation (Fig. 5, 6). Given that the patient was subjected to radiotherapy and immunosuppressive therapy and, besides the characteristics of the disease, the reconstructive surgery was, as it is in most cases, contraindicated for esthetic rehabilitation process, therefore demanding esthetic prosthesis.

\section{Conclusion}

To put it briefly, the manufacturing of nasal prosthesis was presented as the only feasible alternative to the patient's rehabilitation. The prosthesis provided esthetic, comfort to the patient and caused the expected social rehabilitation.

\section{References}

1. Heera R, Choudhary K, Beena VT, Simon R. Strawberry gingivitis: A diagnostic feature of gingival Wegener's granulomatosis! Dent Res J (Isfahan). 2012;9(Suppl 
1):S123-126.

2. Morales-Angulo C, Garcia-Zornoza R, Obeso-Aguera S, Calvo-Alen J, Gonzalez-Gay MA. [Ear, nose and throat manifestations of Wegener's granulomatosis (granulomatosis with polyangiitis)]. Acta Otorrinolaringol Esp. 2012;63(3):206-211.

3. Nishiuma T, Ohnishi H, Yoshimura S, Kinami S, Sakamoto S. A case of granulomatosis with polyangiitis (Wegener's granulomatosis) presenting with marked inflamed tracheobronchial mucosa. Case Rep Med. 2013;2013:208194.

4. Maranhao AS, Chen VG, Rossini BA, Testa JR, Penido Nde O. Mastoiditis and facial paralysis as initial manifestations of Wegener's Granulomatosis. Braz J Otorhinolaryngol. 2012;78(2):80-86.

5. William C, Shiel Jr. Wegener's granulomatosis. Medicine Net.com. 2013 [cited 2013 Nov 12]. Available from: http://www.medicinenet.com/wegeners_granulomatosis/ article.htm.
6. Almouhawis HA, Leao JC, Fedele S, Porter SR. Wegener's granulomatosis: a review of clinical features and an update in diagnosis and treatment. J Oral Pathol Med. 2013;42(7):507-516.

7. Srouji IA, Andrews P, Edwards C, Lund VJ. Patterns of presentation and diagnosis of patients with Wegener's granulomatosis: ENT aspects. J Laryngol Otol. 2007;121(7):653-658.

8. Erickson VR, Hwang PH. Wegener's granulomatosis: current trends in diagnosis and management. Curr Opin Otolaryngol Head Neck Surg. 2007;15(3):170-176.

9. Papadopoulos PJ. Wegener Granulomatosis Medscape reference 2011 [cited 2013 Aug 12]. Available from: http://emedicine.medscape.com/article/332622-overview.

10. Abtahi SM, Omrani M, Karimifar M, Eshaghian A, Kachuei M, Momeni A. Wegener's granulomatosis and mucoromycosis: A case study and review of literature. Adv Biomed Res. 2012;1:23. 